Andrews University

Digital Commons @ Andrews University

Faculty Publications

$1-2020$

\title{
Identifying Active Kelvin-Helmholtz Vortices on Saturn's Magnetopause Boundary
}

B. L. Burkholder

University of Alaska, Fairbanks

P. A. Delamere

University of Alaska, Fairbanks

Jay R. Johnson

Andrews University, jrj@andrews.edu

C.- $\mathrm{s} . \mathrm{Ng}$

University of Alaska, Fairbanks

Follow this and additional works at: https://digitalcommons.andrews.edu/pubs

Part of the Astrophysics and Astronomy Commons

\section{Recommended Citation}

Burkholder, B. L., Delamere, P. A., Johnson, J. R., \& Ng, C.-S. (2020). Identifying active kelvin-helmholtz vortices on saturn's magnetopause boundary. Geophysical Research Letters, 47, e2019GL084206. https://doi.org/10.1029/2019GL084206

This Article is brought to you for free and open access by Digital Commons @ Andrews University. It has been accepted for inclusion in Faculty Publications by an authorized administrator of Digital Commons @ Andrews University. For more information, please contact repository@andrews.edu. 


\section{Geophysical Research Letters}

\section{RESEARCH LETTER \\ 10.1029/2019GL084206 \\ Identifying Active Kelvin-Helmholtz Vortices on Saturn's Magnetopause Boundary}

Key Points:

- Reversed azimuthal flows, likely driven by Kelvin-Helmholtz instabilites, occur dominantly in the dawn-subsolar region

- Many identified Kelvin-Helmholtz events show large turbulent heating, agreeing with $\sim 10^{-15} \mathrm{~W} / \mathrm{m}^{3}$ from hybrid simulations

- Mass diffusion in Kelvin-Helmholtz simulations can be $10^{10} \mathrm{~m}^{2} / \mathrm{s}$, suggesting significant transport events

Correspondence to:

B. L. Burkholder,

blburkholder@alaska.edu

\section{Citation:}

Burkholder, B. L., Delamere, P. A., Johnson, J. R., \& Ng, C.-S. (2020). Identifying active kelvin-helmholtz vortices on saturn's magnetopause boundary. Geophysical Research Letters, 47, e2019GL084206. https:// doi.org/10.1029/2019GL084206

Received 20 JUN 2019 Accepted 27 DEC 2019 Accepted article online 3 JAN 2020

(C)2020. American Geophysical Union. All Rights Reserved.

\author{
B. L. Burkholder' ${ }^{1}$ P. A. Delamere ${ }^{1}$, J. R. Johnson ${ }^{2}$, and C.-S. Ng $^{1}$ \\ ${ }^{1}$ Geophysical Institute, University of Alaska Fairbanks, FairbanksAlaska, USA, ${ }^{2}$ Department of Engineering and \\ Computer Science, Andrews University, Berrien Springs, Michigan, USA
}

\begin{abstract}
For 2, 000 Cassini magnetopause encounters, we analyse plasma and magnetic fields. The boundary can be unstable to the Kelvin-Helmholtz instability (KHI), which can drive large-scale flows identifiable in plasma measurements. Bulk flow reversed from the expected direction near the magnetopause can indicate vorticity associated with active $\mathrm{KH}$, and events are found dominantly in the dawn-subsolar region. KHIs are also responsible for magnetic field fluctuations, and hybrid simulations indicate heating, and transport is significant in an actively growing vortex. Cassini observations are filtered for disturbed magnetic fields near the magnetopause, similar to the signatures from hybrid simulations, with significant fluctuation and current sheet crossings. We also find that these occur most frequently in the dawn-subsolar region. A turbulent heating rate density and mass diffusion coefficient are calculated for these disturbed events and compared with the hybrid simulation to test whether enhanced values for these quantities can identify active $\mathrm{KH}$ events.
\end{abstract}

Plain Language Summary Ocean waves occur when even a light breeze blows over the surface of water. Without wind, the air-water interface would be flat, but strong gusts initiate circulating motion, producing the characteristic breaking wave shape. Similar waves occur anywhere two fluids flowing in different directions are in contact. In space plasmas, this situation is common where solar wind flows past a magnetized body. At Saturn, strong internal magnetic fields produce a cavity in the solar wind, which is filled with plasma by Enceladus. This plasma rotates with the planet, leading to asymmetry in the system, where solar wind flows opposite the magnetospheric flow on the dawn side of the planet, and solar wind flows in the same direction as magnetospheric flow on the dusk side. Previous studies have confoundingly concluded that these waves are most prevalent in the dusk sector, but this is due to the difficulty of observing waves on the dawn magnetopause. The difference being waves on the dawn magnetopause quickly roll-up and "break" similarly to ocean waves, while on the dusk side they keep their structure and move across the magnetopause. We resolve the long-standing conundrum from a comprehensive analysis of the plasma near Saturn's magnetopause boundary.

\section{Introduction}

Solar wind interactions with Saturn's magnetodisc involve asymmetric flow shear between the subcorotating outer magnetosphere and magnetosheath. Flow shear is the primary suspect for tangential drag coupling the solar wind and magnetosphere in a viscous-like process (Axford \& Hines, 1961) rather than large-scale reconnection (Dungey, 1961). Contrasting with Earth, viscous-like interactions have been suggested as dominant at Saturn compared to global-scale reconnection (Masters, 2018). Such analytical considerations (Masters, 2017) rely on characteristic timescales of solar wind variability.

Kelvin-Helmholtz instabilities (KHI) mix plasmas (Hasegawa et al., 2004; Ma et al., 2017; Otto \& Fairfield, 2000), drive small-scale, patchy magnetic reconnection (Ma et al., 2014) and transport momentum across the magnetopause (Burkholder et al., 2017; Delamere et al., 2018). The boundary width is typically $\sim 1 \mathrm{R}_{S}$ (Saturn radius) (Masters et al., 2011) but varies, which is important as flow shear magnitude $(\Delta v)$ determines growth rate (Miura \& Pritchett, 1982). Ma et al. (2015) showed that dawn magnetopause growth rates are large enough that a diffuse boundary layer forms on the order of minutes. $\mathrm{KH}$ vortices induce turbulence through secondary instabilities (Matsumoto \& Hoshino, 2004), which can dissipate magnetic field energy (Saur, 2004). A turbulent cascade has been demonstrated in hybrid simulations of KHI (Delamere et al., 2018), where three-dimensional effects were particularly important. 


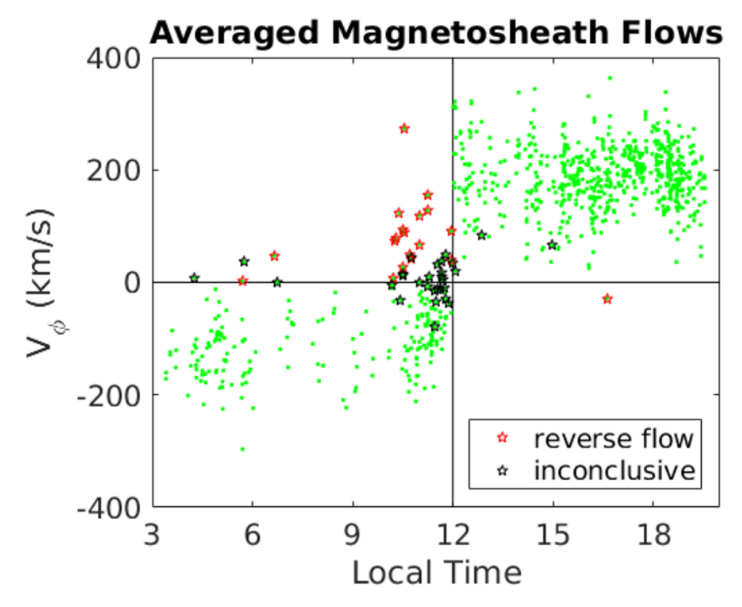

(a)

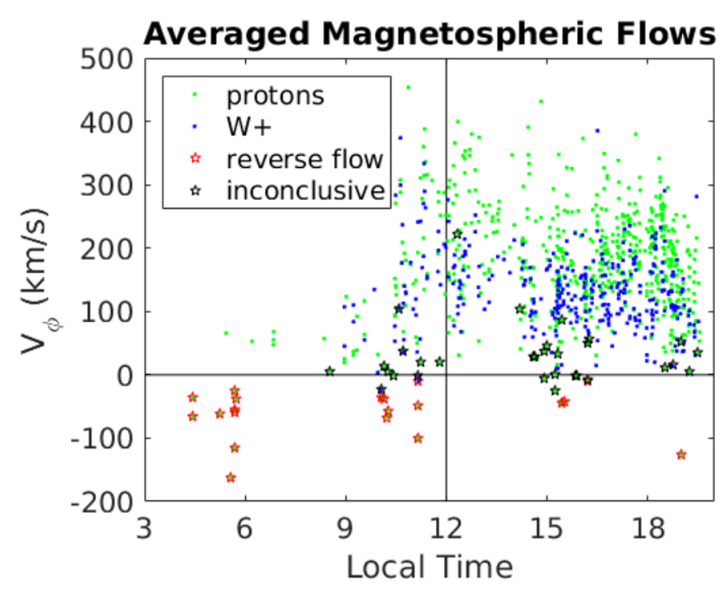

(b)

Figure 1. Magnetosheath (a) and magnetosphere (b) azimuthal flow $\left(v_{\phi}\right)$ averaged over 100-min nearest magnetopause boundary crossings.

Attempts to identify KH at Saturn's magnetopause include boundary normal analysis (Masters et al., 2009) and bipolar magnetic field signatures (current sheets) (Delamere et al., 2013). Surprisingly, the dusk flank (minimum flow shear) was more apt to exhibit KH signatures. Ma et al. (2015) demonstrated that quasi-static $\mathrm{KH}$ structures are indeed prevalent postnoon due to advection from the prenoon sector. The goal here is to identify actively growing (i.e., nonlinear roll-up phase, with large-scale vorticity but precluding the formation of a diffuse boundary layer) $\mathrm{KH}$ vortices at Saturn's magnetopause boundary.

Values for a turbulent heating rate density $q$ and diffusive transport coefficient $D_{\perp}$ are substantial in hybrid simulations of KHI during the active nonlinear growth phase. We are therefore motivated to use Cassini magnetometer (MAG) (Dougherty et al., 2004) data to estimate $q$ and $D_{\perp}$ within KHIs. In addition, we use numerical moments (Thomsen \& Delapp, 2005; Thomsen et al., 2010; Wilson et al., 2008) of the Cassini plasma spectrometer (CAPS) to identify reversed flows as another signature of active KH. With magnetopause boundary identifications from Delamere et al. (2013), we determine the local time (LT) distribution and occurrence rate of active KHIs near Saturn's magnetopause.

\section{Magnetopause Boundary Plasma Flows}

During the active nonlinear roll-up phase of KHI, plasma circulates vortically. Magnetosheath plasma moving toward the subsolar point suggests flow reversal observed within active KH. For magnetospheric plasma at the magnetopause, flow reversed from corotation is likely swirling within a $\mathrm{KH}$ vortex. Interchange motions and other density fluctuations could also be responsible for magnetospheric structure near the boundary (Goertz, 1983), as the specifics of internal transport are not well known.

Delamere et al. (2013) identified 1,919 magnetopause crossings during 2004-2012, mostly occurring near $0^{\circ}$ latitude but $\sim 10 \%$ of which occur $10^{\circ}$ or greater off the equatorial plane. Figure 1a gives average azimuthal velocity $\left(v_{\phi}\right)$ in the magnetosheath from CAPS at these magnetopause encounters. Data are numerical ion moments from CAPS energy spectrograms (http://www.caps.lanl.gov/moments.html). CAPS' field-of-view is important, and we use the filtering proposed by Burkholder et al. (2017). Averages are calculated for magnetosheath data within $100 \mathrm{~min}$ of the crossing. For a static boundary, $100 \mathrm{~min}$ is $0.5 \mathrm{R}_{\mathrm{s}}$ for $5-\mathrm{km} / \mathrm{s}$ spacecraft velocity, chosen because expected scales for $\mathrm{KH}$ vortices are $\sim 1 \mathrm{R}_{s}$. Conversely, 100 min gives $8 \mathrm{R}_{s}$ for a magnetopause expanding at the fast mode speed $C_{f} \sim 80 \mathrm{~km} / \mathrm{s}$. Results are insensitive to the time interval because most magnetosheath encounters are $\leq 200 \mathrm{~min}$. In Figure 1a, red stars indicate when average magnetosheath flow was reversed from tailward. In Figure $1 \mathrm{~b}$, average $v_{\phi}$ for magnetopause traversals is given for protons and $\mathrm{W}^{+}$(sum of water group ions) in the magnetosphere. The average occurs for the same interval as Figure 1a. Red stars indicate when flow was reversed from corotation. Black stars show measurements such that the standard deviation of averaged data gives an inconclusive flow direction.

Given the nearly equal number of inconclusive reversed and expected direction flows, the following statistics apply to absolute averages. Figure 1a gives 31 magnetosheath events with one event postnoon and 
the remainder prenoon. Most reversed flows occur from 10 to 12 LT. Figure 1b shows 25 magnetospheric reversed flow events. The dawn sector shows 16 events, and nine events occur LT $>12$. This gives a KH active dawn magnetopause $14 \pm 2.8 \%$ of the time and $1 \pm 1.4 \%$ for the dusk. The LT sector $10-12$ is active $18 \pm 3.7 \%$ of the time, where flow shear is maximized, and stationary $\mathrm{KH}$ vortices can form due to balanced momentum flux. Interestingly, this estimate for the $\mathrm{KH}$ occurrence rate is similar to 19\% determined at Earth (Kavosi \& Raeder, 2015).

Due to momentum flux balance, the down-tail advection for active $\mathrm{KH}$ should be $\sim \Delta v / 2$, so that reversed flow observations require the correct trajectory. Since the expected magnitude of magnetosheath velocity is $\sim 200 \mathrm{~km} / \mathrm{s}$ for dawn and dusk flanks (Desroche et al., 2013), cases in Figure 1 with significantly reduced flows could indicate KH activity. Starred points LT $<6$ in Figure 1b may be generated by internal transport, but proximity to the magnetopause $(\leq 100 \mathrm{mins})$ suggests $\mathrm{KH}$ origin.

\section{Heating Rate Density and Diffusion Coefficient}

For discussions of $q$ (turbulent heating rate density) based on weak and strong magnetohydrodynamic turbulence, see $\mathrm{Ng}$ et al. (2018). We take $q$ in the strong turbulence regime:

$$
q=\frac{\delta B_{\perp}^{3} k_{\perp}}{\sqrt{u_{0}^{3} \rho}}
$$

where $\delta B_{\perp}$ is perpendicular magnetic field fluctuation at an inverse scale $k_{\perp}$, a perpendicular wavenumber in the inertial range, and $\rho$ is mass density. At Jupiter and Saturn, $q$ has been calculated using a formalism for strong kinetic Alfvén wave (KAW) turbulence and magnetohydrodynamic turbulence (Kaminker et al., 2017; Ng et al., 2018; Saur, 2004; von Papen et al., 2014a). At Saturn, Kaminker et al. (2017) estimates the global average $q \sim 10^{-17} \mathrm{~W} / \mathrm{m}^{3}$.

Diffusive ion transport perpendicular to magnetic field lines was discussed in Johnson and Cheng (1997), and a general form of the diffusion coefficients is presented in equation (1) of (Chaston et al., 2009):

$$
D_{W} \approx \sqrt{\frac{\pi}{8}} \sum_{j=1}^{6} \sum_{k} \frac{1}{\left|k_{||}\right| v_{t i}}\left|\frac{E_{Y}}{B_{0}}\right|^{2} d_{j},
$$

where $k_{\|}$is a parallel wavenumber; $v_{t i}$ is ion thermal velocity; $E_{Y}$ is the transverse electric field fluctuation for wavenumber $k$; and $B_{0}$ is magnetic field strength. The coefficients $d_{1-3}$ correspond to transport contributions from Landau damping, magnetic field gradient drift, and transit time damping, respectively. Coefficients $d_{4-6}$ are cross terms from the coupling of effects described by $d_{1-3}$, which we neglect as they are generally small compared with $d_{1-3}$. Since $d_{1}$ requires the spectrum of electric field fluctuations and $d_{2}$ requires to resolve the magnetic field gradient, we take the limit of small $k_{\perp} \rho_{i}$ where $d_{3}$ dominates since neither measurement can be adequately obtained with Cassini. Furthermore, the integral $R_{11}^{2}$ as defined in Chaston et al. (2009) is a factor in $d_{3}$ and is simplified in this limit:

$$
\lim _{k_{\perp} \rho_{i} \rightarrow 0} R_{11}^{2} \rightarrow k_{\perp}^{2} \rho_{i}^{2} e^{-1 / \beta}
$$

Finally, taking $v_{t i} \sim v_{A}$ (Alfvén velocity) and normalizing in terms of the quantity that controls the transport, which, for the case of $d_{3}$, is the mirror force:

$$
D_{\perp}=\sqrt{\frac{\pi}{2}} \frac{v_{A}}{\left|k_{||}\right|} e^{-1 / \beta} \sum_{k_{\perp} \rho_{i}<1}\left|\frac{\delta B_{||}}{B_{0}}\right|^{2} k_{\perp}^{2} \rho_{i}^{2},
$$

where $\rho_{i}$ is ion gyroradius; $\beta$ is plasma beta (chosen as $\beta=1$ due to superthermal population); and $\delta B_{||} / B_{0}$ is normalized parallel magnetic field fluctuation at an inverse scale $k_{\perp}$. We choose $k_{||}=2 \pi / R_{\text {Saturn }}$ as a lower limit from the scale height of the magnetodisc resonant cavity. Equation (3) quantifies transport from transit time damping (Chaston et al., 2009; Johnson \& Cheng, 1997), which occurs because KAWs drive density and pressure perturbations that produce magnetic field strength fluctuations (magnetic bottles). Resonant particles experience field-aligned acceleration by mirror forces, which results in modified perpendicular drift motion and ion transport across field lines. This KAW-driven mechanism can partially quantify transport, but gyroradius effects, magnetic reconnection, and superdiffusion would not be included. 
Relative magnitudes of $d_{1-6}$ will be the subject of future studies using hybrid simulations given the spectrum of electric field fluctuations. We neglect the other terms in equation (2) for order of magnitude estimates and to facilitate direct comparison between hybrid simulations and Cassini observations.

\section{Hybrid Simulations}

Three-dimensional hybrid simulations (see Delamere et al., 2018 for model description) of the KHI are conducted with Saturn-like parameters (see Table 1 in Delamere et al., 2018). The electron pressure term included in the electron momentum equation captures effects of parallel electric fields associated with KAWs. These results represent initial findings from a study with broader implications for turbulent heating and transport in the hybrid model.

To find $q$, the two-dimensional Fourier power spectrum $P\left(k_{\perp}\right)$ of $\delta B_{\perp}$ is calculated from a slice along the magnetic field. Since $P(k) k \sim \delta b^{2}$ (Leamon et al., 1999), we calculate $\left(P\left(k_{\perp}\right) k_{\perp}\right)^{3 / 2} \sim \delta B_{\perp}^{3}$ to convolve with $k_{\perp}$ in equation (1). Similarly, given the power spectrum $P^{\prime}\left(k_{\perp}\right)$ of $\delta B_{||} / B_{0}$, we calculate $P^{\prime}\left(k_{\perp}\right) k_{\perp} \sim\left|\delta B_{||} / B_{0}\right|^{2}$. Density and temperature for equations (1) and (3) are averaged over the simulation volume, giving $q \sim$ $5 \times 10^{-15} \mathrm{~W} / \mathrm{m}^{3}$ and $D_{\perp} \sim 5 \times 10^{9} \mathrm{~m}^{2} / \mathrm{s}$.

Temporal variation of average energy gives $q \sim 3 \times 10^{-15} \mathrm{~W} / \mathrm{m}^{3}$, a lower limit because $q$ increases with simulation run time. Also note that the grid resolution $(0.4 \times$ ion inertial length) was insufficient to adequately resolve the dissipation scale. The slope of mixed cells versus time gives $D_{\perp} \sim 1 \times 10^{10} \mathrm{~m}^{2} / \mathrm{s}$, where a mixed cell has $75 \%$ particles from one side of the boundary and $25 \%$ from the other (Delamere et al., 2011). This is a factor $\sim 2$ greater than above because magnetic reconnection, ion gyroradius effects, and "superdiffusion" (Cowee et al., 2009) are not quantified by equation (3).

\section{Disturbed Magnetic Fields Near Saturn's Magnetopause}

At Saturn's magnetopause, magnetic reconnection couples planetary and solar wind magnetic fields, generating Maxwell stresses; however, large-scale Dungey-type reconnection has been suggested as a secondary driver compared to viscous interactions (Masters, 2018). Although auroral observations have shown high-shear reconnection between the interplanetary magnetic field (IMF) and planetary magnetic field, it can play a major role (Bunce et al., 2005; Kinrade et al., 2017). KHIs, believed to be a primary component of the viscous interaction, drive intermittent reconnection and produce significant magnetic field fluctuations. A KH active magnetopause will contrast starkly with an inactive boundary. Panels (a) and (b) of Figure 2 give examples of quiet and disturbed magnetic fields observed near Saturn's magnetopause. The global Jupiter magnetosphere simulations show KH activity for all IMF conditions (Zhang et al., 2018), and qualitatively, the global Saturn simulations are similar. While large-scale magnetopause reconnection can be responsible for magnetic field fluctuations, it depends strongly on IMF conditions, such that most events in this paper are likely KH signatures (see also boundary normal analysis by Ma et al., 2015). In addition, large shear flows at the magnetopause suppress classical reconnection (Ma et al., 2016).

With 10-min sliding windows, we analyse 10-s-averaged MAG time series for $>42,000$ windows within $100 \mathrm{~min}$ of Saturn's magnetopause. The choice of $10 \mathrm{~min}$ aligns with expected timescales for magnetic filaments associated with KH (Delamere et al., 2013). All windows come from the magnetospheric side of the magnetopause. Each window is classified active or quiet using three measures of magnetic activity. The threshold between quiet and active is difficult to define; however, the requirement of a current sheet crossing can resolve this ambiguity.

First, the fluctuation $\delta \mathbf{B}=\mathbf{B}(t)-\mathbf{B}_{0}$ is calculated from the magnetic field time series $\mathbf{B}(t)$, where $\mathbf{B}_{0}$ is time-averaged $\mathbf{B}$. The magnetic activity measure is $\delta B=\sqrt{\delta B_{r}^{2}+\delta B_{\theta}^{2}+\delta B_{\phi}^{2}}$ (Kronographic Radial-Theta-Phi coordinates) averaged over the 10-min window. Second, the eigenvalue problem is solved for directions of minimum, intermediate, and maximum variance $n_{\mu}$ :

$$
\sum_{\nu=1}^{3} M_{\mu \nu}^{B} n_{\nu}=\lambda n_{\mu},
$$

where the variance matrix for the magnetic field is given by $M_{\mu \nu}^{B}=\left\langle B_{\mu} B_{\nu}\right\rangle-\left\langle B_{\mu}\right\rangle\left\langle B_{\nu}\right\rangle$ (Paschmann \& Daly, 1998). For eigenvalues $\left[\lambda_{1}, \lambda_{2}, \lambda_{3}\right]$ the geometric mean $\mathcal{E}=\left(\lambda_{1} \lambda_{2} \lambda_{3}\right)^{1 / 3}$ measures total variance for $\mathbf{B}(t)$. 


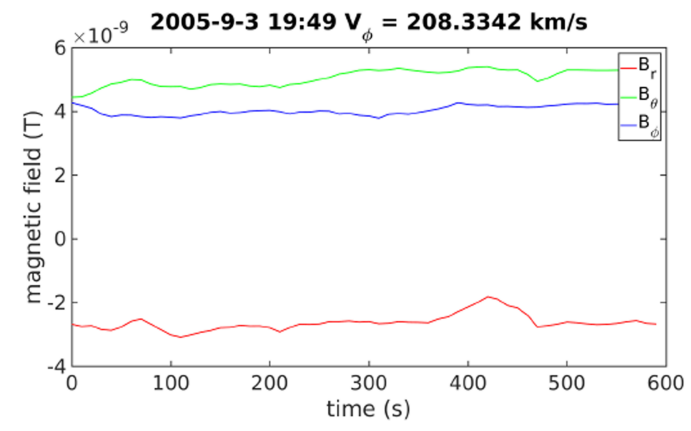

(a)

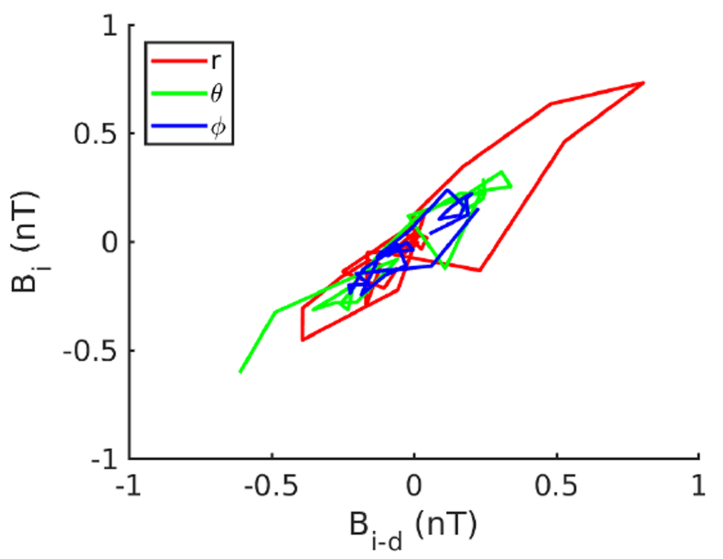

(c)

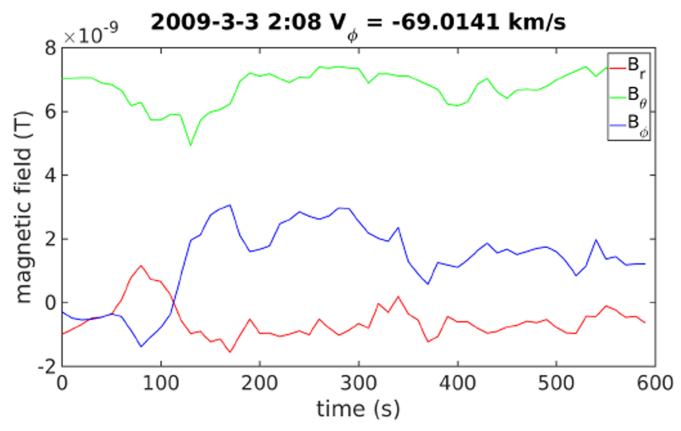

(b)

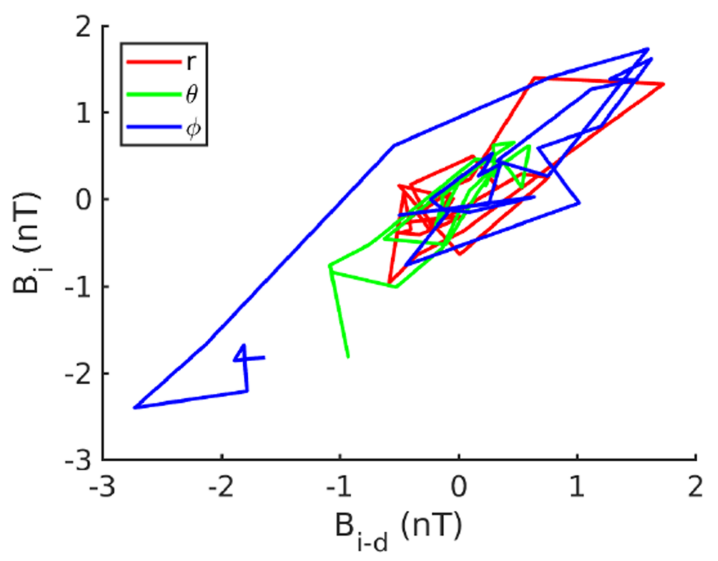

(d)

Figure 2. Quiet (a) and active (b) magnetic fields near Saturn's magnetopause. Note the current sheet crossing in (b) at $t \sim 100$. Panels (c) and (d) show embedding diagrams for (a) and (b), respectively.

Third, a method is adapted from nonlinear time series analysis known as delay coordinate embedding (Bradley \& Kantz, 2015). Embedding diagrams are similar to hodograms; however, two-dimensional hodograms are given in coordinates $x$ and $y$ as $\left(B_{i x}, B_{i y}\right)$ for a common time $i$, while embedding coordinates take the form $\left(B_{i x}, B_{(i-d) x}\right)$ for a common component $x$ where $d$ is the delay length. For $n$-dimensional embedding, $n$ points with temporal separation $d$ become an $n$-vector. Typically, the motivation for time series embedding is to avoid intersections. The $n$ is chosen so the trajectory does not cross itself, and this gives the number of degrees of freedom for the system. The number of degrees of freedom for turbulence would be very large, but fortunately a lower dimensional embedding can be informative. We develop an ad hoc method to determine active versus quiet magnetic fields with $n=2$. Additionally, $d$ is usually chosen from the mutual information or autocorrelation function such that the linear component of the trajectory is neglected. Here $d=10 \mathrm{~s}$ (1-point separation) since quiet conditions exhibit a linear behavior traversing through a steady magnetic field configuration (e.g., Caudal, 1986 force-balanced magnetodisc model) radially increasing or decreasing in strength.

We define a quantity from the embedding for magnetic activity. Many variations associated with a fluctuating time series produce many intersections. The size of the box necessary to enclose the trajectory also carries information about the range of fluctuations. The nonlinear measure of magnetic activity $\mathcal{N}$ sums all intersections individually from $B_{r}, B_{\theta}$, and $B_{\phi}$ to give $\mathcal{N}=$ (number intersections) $* \ell$ where $\ell=\ell_{r 1}+\ell_{r 2}+\ell_{\theta 1}+\ell_{\theta 2}+\ell_{\phi 1}+\ell_{\phi 2}$ is the sum of side lengths for the smallest rectangle enclosing each component's trajectory. Panels (c) and (d) of Figure 2 show the embedding for quiet and active intervals in (a) and (b), respectively.

For numerical thresholds of active versus quiet, histograms of $\log _{10}(\mathcal{E}), \log _{10}(\mathcal{N})$, and $\log _{10}(\delta B)$ are fitted with a Gaussian, although none of the measures for magnetic activity have an exact normal distribution; they are left skewed. The thresholds are one standard deviation to the right of each distribution's peak. This 


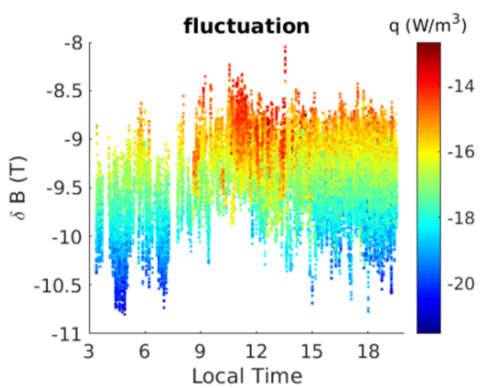

(a)

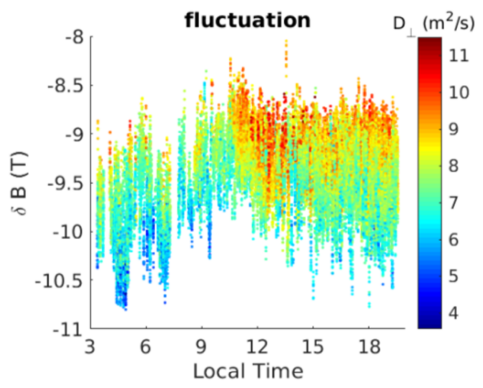

(d)

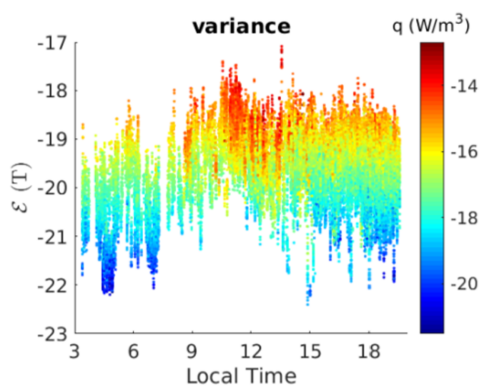

(b)

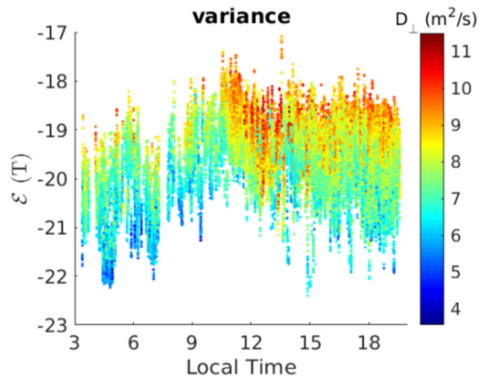

(e)

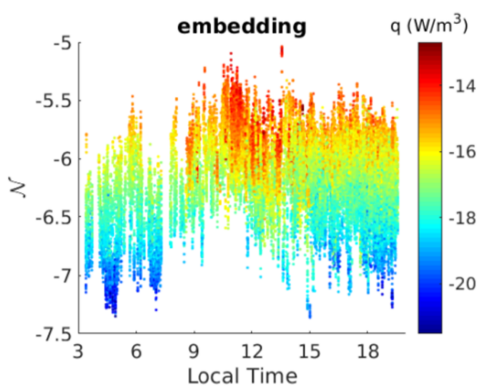

(c)

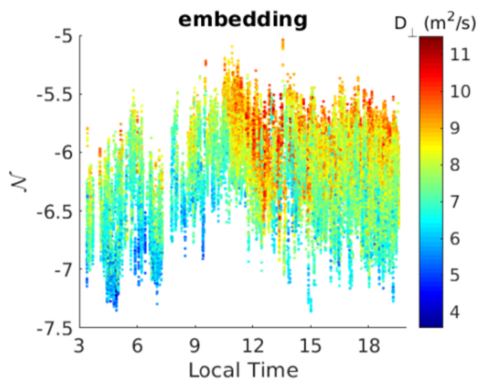

(f)

Figure 3. Measure of magnetic activity versus LT for all windows within $100 \mathrm{~min}$ of magnetopause. Panels (a-c) are colored with $\log _{10} q$, and the other panels (d-f) are colored with $\log _{10} D_{\perp}$. The $y$-axes give $\log _{10} f$ for $f$ the particular measure of magnetic activity.

semi-arbitrary threshold is motivation for choosing multiple measures of magnetic activity to give confidence in choosing windows that lie near the threshold. Finally, the analysis requires at least one current sheet (sign change of two or more magnetic field components) to be labeled a KH active window (Delamere et al., 2011). This gives a boundary with active magnetic fields $3 \pm 0.2 \%$ of the time. With respect to LT active magnetic fields occur near the magnetopause $5 \pm 0.4 \%$ of the time LT $<12,2 \pm 0.3 \%$ of the time LT $>12$, and $12 \pm 0.7 \%$ between LT 10 and 12 . Also, note the value for $v_{\phi}$ given in Figures $2 \mathrm{a}$ and $2 \mathrm{~b}$, which was measured by CAPS during the 10-min window. While it would seem prudent to correlate active magnetic fields with reversed flows, it is difficult to carry out this analysis since the 7-min actuation time for CAPS means that it is impossible to capture variability in the flow on shorter timescales (see Wilson et al., 2012).

\subsection{Cassini Heating Rate Density}

Cassini's observed $q$ is approximated with $k_{\perp}=2 \pi f /\left[v_{r e l} \sin \left(\theta_{v B}\right)\right]$ as given by von Papen et al. (2014b), where $f$ is the frequency range resolved in the power spectrum; $\theta_{v B}$ is the angle between flow and magnetic field; and $v_{r e l}$ is the flow velocity relative to Cassini. These velocities can be determined using an empirically fitted radial profile (Thomsen et al., 2010). For a given 1-s-averaged MAG time series, the one-dimensional power spectrum $P(f)$ of $\delta B_{\perp}$ is calculated with a Morlet wavelet transform (Tao et al., 2015). Similarly to section $4,(P(f) f)^{3 / 2} \sim \delta B_{\perp}^{2}$ is integrated with a factor $k_{\perp}$, as in equation (3). The remaining factor $1 / \sqrt{\mu_{0}^{3} \rho}$ gives $q$, with density determined by an empirical profile (Thomsen et al., 2010).

Panels (a-c) of Figure 3 show magnetic activity LT distributions colored by $q$ for magnetospheric windows within $100 \mathrm{~min}$ of the magnetopause. There are more points compared to Figure 1 because MAG data availability is far better than CAPS, and every window is shown rather than averages. The $q$ distributions are similar, indicating thatthe methods agree well. Each measure shows a clear trend of larger $q$ for larger measure of magnetic activity, and a significant hot spot from LT $\sim 10-13$.

Histograms in Figure 4a show distributions of $q$ near (100 mins) Saturn's magnetopause. The dawn and dusk sectors both have geometric mean $q \sim 2 \times 10^{-17} \mathrm{~W} / \mathrm{m}^{3}$, while active events have geometric mean $q \sim 1 \times 10^{-15} \mathrm{~W} / \mathrm{m}^{3}$. The broader dawn distribution suggests asymmetry in the heating characteristics on the dawn versus dusk magnetopause. The red axis in Figure 4a shows the geometric mean of $q$ as a function of time from magnetopause crossing. Average $q$ maximizes nearest the boundary and decreases moving away. 


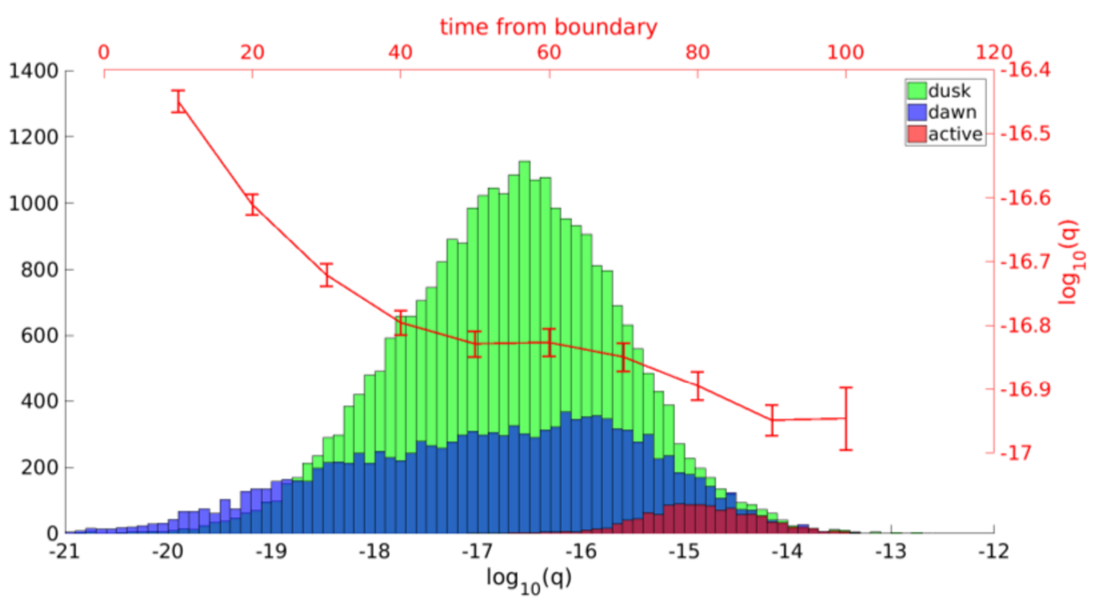

(a)

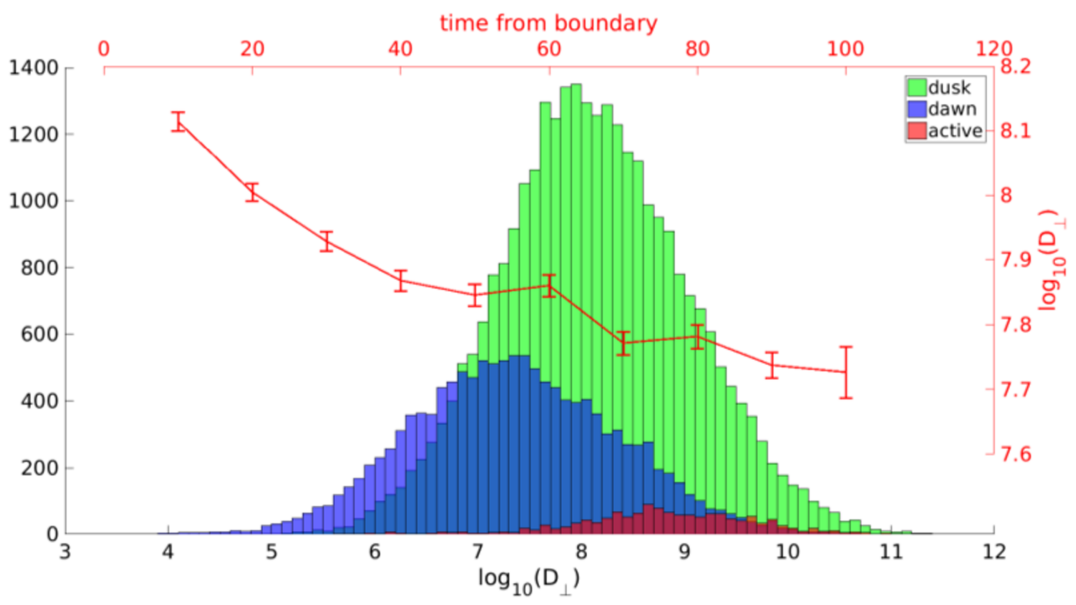

(b)

Figure 4. Black axes give histograms of $q$ (a) and $D_{\perp}$ (b) for dawn (blue), dusk (green), and active windows (red) within $100 \mathrm{~min}$ of the magnetopause. Red axes show average with time from magnetopause crossing over all LT.

To estimate the relevance of these events for the global energy budget, consider that plasma parcels near the subsolar magnetopause boundary take $\sim 6 \mathrm{hr}$ before being swept down tail to exit the magnetosphere. Given $q \sim 10^{-15} \mathrm{~W} / \mathrm{m}^{3}$ for $6 \mathrm{hr}$, plasma temperature can change by $100 \mathrm{~s}$ of eV for average Saturn parameters. This is a significant amount of heating given typical temperatures in the outer magnetosphere $\left(10^{2}-10^{3} \mathrm{eV}\right.$; Thomsen et al., 2010). However it is important to note these events are an episodic spot heating. As a consequence of ideal flux circulation, it may also be true that magnetic fluctuations dissipate in the magnetosphere, while plasma escapes to the solar wind.

\subsection{Cassini Diffusion Coefficient}

To calculate $D_{\perp}$ in equation (3), one-dimensional Fourier power spectra $P^{\prime}(f)$ of $\delta B_{\|} / B_{0}$ are derived from 1-s-averaged MAG time series. As in section $4, P^{\prime}(f) f \sim\left|\delta B_{||} / B_{0}\right|^{2}$. Density and temperature profiles from Thomsen et al. (2010) give $v_{A}$ and $\rho_{i}$. Panels (d-f) of Figure 3 show the LT distribution of magnetic activity colored by $D_{\perp}$ for magnetospheric windows within $100 \mathrm{~min}$ of the magnetopause. The distributions are similar for each measure of magnetic activity, and they are symmetric in the vicinity of the subsolar point, while the flanks are asymmetric with more large values found in the dusk. However, for LT $<12,2 \%$ of events are active and have $D_{\perp}>1 \times 10^{9}$, while for the dusk only $1 \%$ are so, and $4 \%$ for the prenoon sector LT 10-12. There is a trend of larger $D_{\perp}$ for larger measure of magnetic activity, similarly to results for $q$.

Figure $4 \mathrm{~b}$ shows histograms of $D_{\perp}$ near Saturn's magnetopause, similar to Figure fig:q_histsa. There is LT asymmetry, with dusk geometric mean $D_{\perp} \sim 1 \times 10^{8} \mathrm{~m}^{2} / \mathrm{s}$ and dawn geometric mean $D_{\perp} \sim 2 \times 10^{7}$. The active events have geometric mean $D_{\perp} \sim 7 \times 10^{8} \mathrm{~m}^{2} / \mathrm{s}$. It is interesting that many large values for $D_{\perp}$ are 
not included in the active events, suggesting that the chosen measures for magnetic activity do not capture a strong dependence on the parallel magnetic field fluctuation. Such fluctuations in magnetic field strength may naturally occur in the high beta plasma near Saturn's magnetopause. As in panel (a), the red axis for panel (b) shows average $D_{\perp}$ versus time from magnetopause boundary, which decreases moving away from the boundary. Again it is important to note that enhanced transport events would be episodic. Islands of magnetospheric $W^{+}$plasma would be formed in the magnetosheath as found $15 \%$ of the time by Sergis et al. (2013), which agrees with the estimated occurrence rates of active $\mathrm{KH}$ in the prenoon sector from sections 2 and 5 .

An estimate for mass transported through the boundary with $D_{\perp} \sim 10^{9} \mathrm{~m}^{2} / \mathrm{s}$ can be obtained from the diffusion equation. The empirical density profile from Thomsen et al. (2010) can approximate $\nabla^{2} n$ at $R=25 R_{\text {Saturn }}$. This is likely a bad approximation to the magnetopause density gradient, particularly within active $\mathrm{KH}$ but gives a zeroth order approximation. The resulting $\frac{\partial n}{\partial t}=0.62 \mathrm{~m}^{3} / \mathrm{s} \mathrm{integrated} \mathrm{over} \mathrm{a} \mathrm{vol-}$ ume approximating the dawn-side boundary layer gives a transport rate $\sim 0.1 \mathrm{~kg} / \mathrm{s}$. This is only $0.2 \%$ of the $55 \mathrm{~kg} / \mathrm{s}$ escaping the magnetosphere on average (Neupane et al., 2019). Using the upper limit $D_{\perp} \sim 10^{10} \mathrm{~m}^{2} / \mathrm{s}$ from hybrid simulations the transport rate is still only $1 \mathrm{~kg} / \mathrm{s}$, indicating the dominant region of mass loss is down tail. Although, the global mass budget may still depend on KHIs since they could play a role in the nonlinear development of instabilities with much sharper gradients. In future work we will obtain a more quantitatively complete estimation of the mass transport, both from neglected terms in equation (2) and also transport associated with magnetic reconnection, large ion gyroradius effects, and superdiffusion.

\section{Conclusions}

Since Saturn's boundary layer width (and associated magnetopause $\Delta v$ ) varies, active KH vortices may be rarely observed. The dawn magnetopause has large $\mathrm{KH}$ growth rates so the boundary quickly diffuses into a broad layer with an uncertain lifetime. The dusk magnetopause is mostly structured by nonevolving $\mathrm{KH}$ waves advecting downtail rather than actively rolling-up (Ma et al., 2015; Zhang et al., 2018). We summarize our findings as follows:

- Reversed flows near Saturn's magnetopause indicate active KH vortices $\sim 14 \%$ of the time prenoon and $\sim 1 \%$ postnoon. A majority of prenoon events occur in the LT sector 10-12 ( 18\% occurrence rate) where there can be a stationary $\mathrm{KH}$ vortex.

- Theoretical values for $q$ and $D_{\perp}$ in hybrid simulations of the KHI using equations (1) and 3 are $q \sim$ $10^{-15} \mathrm{~W} / \mathrm{m}^{3}$ and $D_{\perp} \sim 10^{9} \mathrm{~m}^{2} / \mathrm{s}$ for typical Saturn parameters.

- Active magnetic fields with current sheet crossings are found on the magnetospheric side of the magnetopause boundary $\sim 2 \%$ of the time L $>12, \sim 5 \%$ for LT $<12,12 \%$ from LT 10-12, and $3 \%$ globally. While magnetic fluctuations may also be associated with magnetopause reconnection, their abundance in the region of maximum flow shear suggests the majority have a $\mathrm{KH}$ origin.

- Active magnetic fields can be associated with $q$ and $D_{\perp}$ comparable to the hybrid simulation, particularly at LT $10-12$.

- Values of $q$ and $D_{\perp}$ significantly above the background allows a possible identification of KH active intervals; however, the high beta environment renders this method imprecise due to background magnetic field fluctuations.

- The hot spot for large $q$ and $D_{\perp}$ in the subsolar region as well as prominence of reversed flows in the LT sector 10-12 resolves the conundrum of the dawn-versus-dusk prevalence of KH waves and confirms the findings of Ma et al. (2015).

\section{Acknowledgments}

The authors acknowledge support from NASA grant NNX15AH09G. Cassini MAG (https://pds-ppi.igpp. ucla.edu/search/?sc=Cassini\& $\mathrm{t}=$ Saturn\&i=MAG) and CAPS/IMS ion moments (https://pds-ppi.igpp. ucla.edu/search/?t=Saturn\&i=CAPS) are available as peer-reviewed data products at the Planetary Data System. The model source code in this paper can be accessed at https://github.com/ bhstauffer.

\section{References}

Axford, W. I., \& Hines, C. O. (1961). A unifying theory of high latitude geophysical phenomena and geomagnetic storms. Canadian Journal of Physics, 39, 1433-1464.

Bradley, E., \& Kantz, H. (2015). Nonlinear time-series analysis revisited. Chaos: An Interdisciplinary Journal of Nonlinear Science, 25(9), 97-610. https://doi.org/10.1063/1.4917289

Bunce, E., Cowley, S. W. H., \& Milan, S. (2005). Interplanetary magnetic field control of Saturn's polar cusp aurora. Annales Geophysicae, 23, 1405-1431. https://doi.org/10.5194/angeo-23-1405-2005

Burkholder, B., Delamere, P. A., Ma, X., Thomsen, M. F., Wilson, R. J., \& Bagenal, F. (2017). Local time asymmetry of Saturn's magnetosheath flows. Geophysical Research Letters, 44, 5877-5883. https://doi.org/10.1002/2017GL073031

Caudal, G. (1986). A self-consistent model of Jupiter's magnetodisc including the effects of centrifugal force and pressure. Journal of Geophysical Research, 91, 4201-4221. https://doi.org/10.1029/JA091iA04p04201 
Chaston, C. C., Johnson, J. R., Wilber, M., Acuna, M., Goldstein, M. L., \& Reme, H. (2009). Kinetic Alfvén wave turbulence and transport through a reconnection diffusion region. Physical Review Letters, 102, 15001. https://doi.org/10.1103/PhysRevLett.102.015001

Cowee, M. M., Winske, D., \& Gary, S. P. (2009). Two-dimensional hybrid simulations of superdiffusion at the magnetopause driven by Kelvin-Helmholtz instability. Journal of Geophysical Research, 114, A10209. https://doi.org/10.1029/2009JA014222

Delamere, P. A., Burkholder, B., \& Ma, X. (2018). Three-dimensional hybrid simulation of viscous-like processes at Saturn's magnetopause boundary. Geophysical Research Letters, 45, 7901-7908. https://doi.org/10.1029/2018GL078922

Delamere, P. A., Wilson, R. J., Eriksson, S., \& Bagenal, F. (2013). Magnetic signatures of Kelvin-Helmholtz vortices on Saturn's magnetopause: Global survey. Journal of Geophysical Research: Space Physics, 118, 393-404. https://doi.org/10.1029/2012JA018197

Delamere, P. A., Wilson, R. J., \& Masters, A. (2011). Kelvin-helmholtz instability at Saturn's magnetopause: Hybrid simulations. Journal of Geophysical Research, 116, A10222. https://doi.org/10.1029/2011JA016724

Desroche, M., Bagenal, F., Delamere, P. A., \& Erkaev, N. (2013). Conditions at the magnetopause of Saturn and implications for the solar wind interaction. Journal of Geophysical Research:Space Physics, 118, 3087-3095. https://doi.org/10.1002/jgra.50294

Dougherty, M. K., Kellock, S., Southwood, D. J., Balogh, A., Smith, E. J., Tsurutani, B. T., et al. (2004). The Cassini magnetic field investigation. Space Science Reviews, 114, 331-383. https://doi.org/10.1007/s11214-004-1432-2

Dungey, J. W. (1961). Interplanetary magnetic field and the auroral zones. Physical Review Letters, 6(2), 47-48. https://doi.org/10.1103/ PhysRevLett.6.47

Goertz, C. K. (1983). Detached plasma in Saturn's front side magnetosphere. Geophysical Research Letters, 10(6), 455-458. https://doi.org/ 10.1029/GL010i006p00455

Hasegawa, H., Fujimoto, M., Phan, T., Reme, H., Balogh, A., Dunlop, M., et al. (2004). Transport of solar wind into Earth's magnetosphere through rolled-up Kelvin-Helmholtz vortices. NATURE, 430, 755-758. https://doi.org/10.1038/nature02799

Johnson, J. R., \& Cheng, C. Z. (1997). Kinetic Alfvén waves and plasma transport at the magnetopause. Geophysical Research Letters, 24, 1423-1426. https://doi.org/10.1029/97GL01333

Kaminker, V., Delamere, P. A., Ng, C. S., Dennis, T., Otto, A., \& Ma, X. (2017). Local time dependence of turbulent magnetic fields in Saturn's magnetodisc. Journal of Geophysical Research: Space Physics, 122, 3972-3984. https://doi.org/10.1002/2016JA023834

Kavosi, S., \& Raeder, J. (2015). Ubiquity of Kelvin Helmholtz waves at Earth's magnetopause. Nature Communications, https://doi.org/10. $1038 /$ ncomms 8019

Kinrade, J., Badman, S. V., Bunce, E. J., Tao, C., Provan, G., Cowley, S. W. H., et al. (2017). An isolated, bright cusp aurora at saturn. Journal of Geophysical Research: Space Physics, 122, 6121-6138. https://doi.org/10.1002/2016JA023792

Leamon, R. J., Smith, C. W., Ness, N. F., \& Wong, H. K. (1999). Dissipation range dynamics: Kinetic Alfvén waves and the importance of $\beta_{e}$. Journal of Geophysical Research, 104, 22,331-22,344. https://doi.org/10.1029/1999JA900158

Ma, X., Delamere, P., Otto, A., \& Burkholder, B. (2017). Plasma transport driven by the three-dimensional Kelvin-Helmholtz instability. Journal of Geophysical Research: Space Physics, 122, 10,382-10,395. https://doi.org/10.1002/2017JA024394

Ma, X., Otto, A., \& Delamere, P. A. (2014). Interaction of magnetic reconnection and Kelvin-Helmholtz modes for large magnetic shear: 1. Kelvin-helmholtz trigger. Journal of Geophysical Research: Space Physics, 119, 781-797. https://doi.org/10.1002/2013JA019224

Ma, X., Otto, A., \& Delamere, P. A. (2016). Magnetic reconnection with a fast perpendicular sheared flow. Journal of Geophysical Research: Space Physics, 121, 9427-9442. https://doi.org/10.1002/2016JA023107

Ma, X., Stauffer, B., Delamere, P. A., \& Otto, A. (2015). Asymmetric Kelvin-Helmholtz propagation at Saturn's dayside magnetopause. Journal of Geophysical Research: Space Physics, 120, 1867-1875. https://doi.org/10.1002/2014JA020746

Masters, A. (2017). Model based assessments of magnetic reconnection and Kelvin Helmholtz instability at Jupiter's magnetopause. Journal of Geophysical Research: Space Physics, 122, 11,154-11,174. https://doi.org/10.1002/2017ja024736

Masters, A. (2018). A more viscous-like solar wind interaction with all the giant planets. Geophysical Research Letters, 45, $7320-7329$. https://doi.org/10.1029/2018GL078416

Masters, A., Achilleos, N., Bertucci, C., Dougherty, M. K., Kanani, S. J., Arridge, C. S., et al. (2009). Surface waves on Saturn's dawn flank magnetopause driven by the Kelvin-Helmholtz instability. Planetery and Space Science, 57, 1769-1778. https://doi.org/10.1016/j.pss. 2009.02.010

Masters, A., Mitchell, D. G., Coates, A. J., \& Dougherty, M. K. (2011). Saturn's low-latitude boundary layer: 1. Properties and variability. Journal of Geophysical Research, 116, A06210. https://doi.org/10.1029/2010JA016421

Matsumoto, Y., \& Hoshino, M. (2004). Onset of turbulence induced by a Kelvin-Helmholtz vortex. Geophysical Research Letters, 31 , L02807. https://doi.org/10.1029/2003GL018195

Miura, A., \& Pritchett, P. L. (1982). Nonlocal stability analysis of the MHD Kelvin-Helmholtz instability in a compressible plasma. Journal of Geophysical Research, 87(A9), 7431-7444. https://doi.org/10.1029/JA087iA09p07431

Neupane, B. R., Delamere, P. A., Wilson, R. J., \& Ma, X. (2019). Quantifying mass and magnetic flux transport in Saturn's magnetosphere. Journal of Geophysical Research: Space Physics, 124, 1916-1926. https://doi.org/10.1029/2018JA026022

Ng, C. S., Delamere, P. A., Kaminker, V., \& Damiano, P. A. (2018). Radial transport and plasma heating in Jupiter's magnetodisc. Journal of Geophysical Research: Space Physics, 123, 6611-6620. https://doi.org/10.1029/2018JA025345

Otto, A., \& Fairfield, D. H. (2000). Kelvin-Helmholtz instability at the magnetotail boundary: MHD simulation and comparison with Geotail observations. Journal of Geophysical Research, 105, 21,175-21,190.

Paschmann, G., \& Daly, P. W. (1998). Analysis Methods for Multi-Spacecraft Data (pp. 185). Noordwijk, Netherlands: ESA Publ. Div.

Saur, J. (2004). Turbulent heating of Jupiter's middle magnetosphere. The Astrophysical Journal, 602, L137-L140. https://doi.org/10.1086/ 382588

Sergis, N., Jackman, C. M., Masters, A., Krimigis, S. M., Thomsen, M. F., Hamilton, D. C., et al. (2013). Particle and magnetic field properties of the Saturnian magnetosheath: Presence and upstream escape of hot magnetospheric plasma. Journal of Geophysical Research: Space Physics, 118, 1620-1634. https://doi.org/10.1002/jgra.50164

Tao, C., Sahraoui, F., Fontaine, D., de Patoul, J., Chust, T., Kasahara, S., \& Retinò, A. (2015). Properties of Jupiter's magnetospheric turbulence observed by the galileo spacecraft. Journal of Geophysical Research: Space Physics, 120, 2477-2493. https://doi.org/10.1002/ 2014JA020749

Thomsen, M. F., \& Delapp, D. M. (2005). Numerical moments computation for CAPS/IMS: Los Alamos National Laboratory.

Thomsen, M. F., Reisenfeld, D. B., Delapp, D. M., Tokar, R. L., Young, D. T., Crary, F. J., et al. (2010). Survey of ion plasma parameters in Saturn's magnetosphere. Journal of Geophysical Research, 115, A10220. https://doi.org/10.1029/2010JA015267

von Papen, M., Saur, J., \& Alexandrova, O. (2014a). Turbulent magnetic field fluctuations in Saturn's magnetosphere. Journal of Geophysical Research: Space Physics, 119, 2797-2818. https://doi.org/10.1002/2013JA019542

von Papen, M., Saur, J., \& Alexandrova, O. (2014b). Turbulent magnetic field fluctuations in Saturn's magnetosphere. Journal of Geophysical Research: Space Physics, 119, 2797-2818. https://doi.org/10.1002/2013JA019542 
Wilson, R. J., Delamere, P. A., Bagenal, F., \& Masters, A. (2012). Kelvin-helmholtz instability at Saturn's magnetopause: Cassini ion data analysis. Journal of Geophysical Research, 117, A03212. https://doi.org/10.1029/2011JA016723

Wilson, R. J., Tokar, R. L., Henderson, M. G., Hill, T. W., Thomsen, M. F., \& Pontius, D. H. (2008). Cassini plasma spectrometer thermal ion measurements in Saturn's inner magnetosphere. Journal of Geophysical Research, 113, A12218. https://doi.org/10.1029/2008JA013486 Zhang, B., Delamere, P. A., Ma, X., Burkholder, B., Wiltberger, M., Lyon, J. G., et al. (2018). Asymmetric Kelvin-Helmholtz instability at Jupiter's magnetopause boundary: Implications for corotation-dominated systems. Geophysical Research Letters, 45, 56-63. https://doi. org/10.1002/2017GL076315 